PHYSICAL REVIEW D 89, 027503 (2014)

\title{
Non-Einstein source effects in massive gravity
}

\author{
S. Deser* \\ Lauritsen Lab, Caltech, Pasadena, California 91125, USA and Physics Department, Brandeis University, \\ Waltham, Massachusetts 02454, USA \\ A. Waldron \\ Department of Mathematics, University of California, Davis, California 95616, USA
}

(Received 24 October 2013; published 13 January 2014)

\begin{abstract}
We exhibit novel effects (absent in GR) of sources in massive gravity. First, we show that removing its ghost mode forces a field-current identity: The metric's trace is locally proportional to that of its stress tensor; a point source implies a metric singularity enhanced by the square of the graviton's range. Second, exterior solutions acquire spatial stress hair-their metric components depend on the interior $T_{i j}$. Also, in contrast to naïve expectations, the Newtonian potential of a source is now determined by both its spatial stress and mass. Our explicit results are obtained at linear, Fierz-Pauli, level but qualitatively persist nonlinearly.
\end{abstract}

DOI: $10.1103 /$ PhysRevD.89.027503

PACS numbers: 04.50.Kd, 04.30.Db, 04.50.-h

\section{INTRODUCTION}

Massive gravity (mGR) remains a growth industry (for an early catalog, see Ref. [1]), despite its numerous problems; for example, removing its ghost mode allows faster-than-light, acausal, propagation of the remaining, "physical" ones [2]. Here we exhibit some novel differences from general relativity (GR) in the presence of matter, already at linear level [3]. The first deviation, reminiscent of the old field-current identity (FCI) [4], is that the metric's and stress tensor's traces are locally proportional; thus, a point source produces a naked metric singularity. A second one is the loss of the no-hair theorem: Static, spherically symmetric, conserved, spatial stresses contribute to the exterior metric (not just through the FCI), contrary to bald GR [5]. The FCI effect is enhanced by an enormous factor $m^{-2}$ and becomes singular in the massless limit. Further, mGR's Newtonian potential depends on the source's interior spatial stresses as well as its mass.

In Planck units, the linear, Fierz-Pauli (FP) field equations are

$$
\mathcal{G}_{\mu \nu}:=G_{\mu \nu}^{L}(h)-\frac{1}{2} m^{2}\left(h_{\mu \nu}-\eta_{\mu \nu} h\right)=\frac{1}{2} T_{\mu \nu},
$$

where $G_{\mu \nu}^{L}$ is the linearized Einstein tensor and the mass term is the unique ghost-free combination. We assume conservation of the source $T_{\mu \nu}$ [6]. The usual double divergence of Eq. (1) implies vanishing of the linearized scalar curvature-here the, nongauge fixing, $\square h-\partial . \partial . h$, combination-which means, upon tracing Eq. (1), that

$$
3 m^{2} h=T .
$$

deser@brandeis.edu

†wally@math.ucdavis.edu
This is our FCI. It locally equates the traces of the metric's $h_{\mu \nu}$ and source's $T_{\mu \nu}$ and (except for null sources) is discontinuous in the $m \rightarrow 0$ limit. A point mass thus induces a delta function singularity $M \delta^{3}(r)$ in $h:=h_{\mu}^{\mu}$. Note that even at a finite (physical) mass, there is an enormous enhancement of the metric by the square of mGR's range $\ell^{2}:=m^{-2}$. Furthermore, this is not merely a linearized theory artifact but is inherent to nonlinear, five degrees of freedom (DoF), mGR extensions with algebraic mass terms [7]: There, the left hand side of Eq. (2) is supplemented by terms involving at most first metric derivative, contorsion, terms [2,8-10], so that an FCI persists in full mGR.

\section{DETAILS}

The calculation involves the solution of the sourceful FP equations (1), using the standard massive spin-2 propagator,

$$
\Delta_{\mu \nu \alpha \beta} \sim \frac{P_{\mu(\alpha} P_{\beta) \nu}-\frac{1}{3} P_{\mu \nu} P_{\alpha \beta}}{p^{2}+m^{2}},
$$

defined in terms of the on-shell projectors

$$
P_{\mu \nu}:=\eta_{\mu \nu}+\frac{p_{\mu} p_{\nu}}{m^{2}} .
$$

The essential difference between these projectors and those of massless spin 2 is that the latter involve not $p_{\mu} p_{\nu} / \mathrm{m}^{2}$ but $p_{\mu} p_{\nu} / p^{2}$ : Because the massive one's trace has a $p^{2} / m^{2}$ numerator, it can cancel the propagator's denominator, while the massless one just traces to unity. Using Eq. (3), the metric produced by a conserved $T_{\mu \nu}$ is

$$
h_{\mu \nu}=\Delta_{\mu \nu \alpha \beta} T^{\alpha \beta}=\frac{T_{\mu \nu}-\frac{1}{3} P_{\mu \nu} T}{\square-m^{2}} \Rightarrow h=\frac{T}{3 m^{2}} .
$$

The interaction $\int h_{\mu \nu} t^{\mu \nu}$ that this implies between two sources is not (quite) the usual 


$$
I \sim \int\left[t^{\mu \nu} \frac{1}{\square-m^{2}} T_{\mu \nu}-\frac{1}{3} t \frac{1}{\square-m^{2}} T\right],
$$

because reaching the latter required integrating the $p_{\mu} p_{\nu}$ term of $P_{\mu \nu}$ in Eq. (3) by parts, thereby implicitly losing the FCI term: While indeed $\partial \cdot t_{\nu}=\partial \cdot T_{\nu}=0$, the trace part $p^{2} / m^{2}$ of $P_{\mu \nu}$ has been lost in the process. (In the massless case, there is no ambiguity since tracing $p_{\mu} p_{\nu} / p^{2}$ yields unity.) This new contribution, $\delta I$, to the interaction is proportional to $t_{\alpha}^{\alpha} h$; hence, by Eq. (2),

$$
\delta I \sim \int t h=\frac{1}{3 m^{2}} \int t T .
$$

This is a contact interaction between two (non-null) sources, so not really observable macroscopically, but it might be seen in their s-wave scattering-which would be enormously magnified by the square of mGR's range. To be sure, linearized theory misses any horizonlike features of the full nonlinear extension, so we cannot make realistic estimates of the effect. Note that the full $T_{\mu \nu}$ 's trace is involved, so already here we see the presence of hair; i.e., interior, spatial, $T_{i j}$ components, even of spherically symmetric sources, contribute. [Since we are working at linear level, we can treat this source independently of any $T_{00}(r)$.] Turning now to the hair effect itself, we must consider a local, spherically symmetric, static, conserved source $T_{i j}(r)$. Hence, by conservation, this $T_{i j}$ must be the transverse projector of some scalar [11]. The naïve ansatz $T_{i j}=\frac{1}{2}\left(\delta_{i j}-\partial_{i} \partial_{j} \Delta^{-1}\right) T(r)$ is disallowed because only its trace is local. Instead, locality can be restored by writing

$$
T(r)=\Delta \tau(r) \rightarrow T_{i i}=\Delta \tau,
$$

for some local $\tau(r)$. Such sources are not physical in GR because they yield no external interactions (see below), thereby upholding the traditional no-hair lore. As we shall see, the situation in mGR is rather different.

Inserting the source (4) into Eq. (3), in terms of the Yukawa potential $Y:=\left(\Delta-m^{2}\right)^{-1}$, one finds

$$
\begin{aligned}
h_{i j} & =\int \mathrm{d}^{3} x Y\left[T_{i j}-\frac{1}{3}\left(\delta_{i j}+\frac{p_{i} p_{j}}{m^{2}}\right) T\right], \\
h_{00} & =\frac{1}{3} \int \mathrm{d}^{3} x Y T
\end{aligned}
$$

[for which the trace $h=-h_{00}+h_{i i}$ indeed obeys the FCI (2)]. Hence, mGR's Newtonian potential $h_{00}$ depends on spatial stresses. More precisely, when the interior $\tau$ and exterior $t_{00}$ do not overlap, their hairy Newtonian interaction is

$$
I \sim \int t_{00} Y \Delta \tau=\int t_{00}\left[1+m^{2} Y\right] \tau=m^{2} \int t_{00} Y \tau .
$$

While nonvanishing, this new interaction is suppressed by the product $(m R)^{2}$ where $R$ is the radius of the source [12]. Similarly, the spatial stress interaction is $\int t^{i j} h_{i j} \sim$ $-\frac{1}{3} m^{2} \int t_{i i} Y \tau$. The third possible static conserved source $T_{0 i}=\varepsilon_{i j k} \partial_{j} J_{k}$ generates $h_{0 i}$, leading to a spin-spin interaction, since $T_{0 i}(r) \sim \epsilon_{i j k} \partial_{j} J_{k} \delta^{3}(r)$ is both local and conserved. The resulting interactions are identical to the textbook Maxwell magnetic dipole-dipole's, including a $\vec{j} \cdot \vec{j}$ contact term. In mGR there are forces beyond Maxwellian ones, since the derivatives in the momentum densities now act on $Y$, rather than just $C$ [13]; there is also a novel Yukawa term $\sim m^{2} \int j Y J$.

\section{CONCLUSION}

Using the linearized, FP, approximation to $\mathrm{mGR}$, we found novel properties of mGR's sourceful solutions that deviate from GR-based exterior solution expectations [14]. While these were obtained explicitly at linear level, they should persist qualitatively in full mGR. Specifically, we found the following. (i) The scalar constraint is proportional to $\mathrm{m}^{-2}$ times the trace of the source $T_{\mu \nu}$-in particular, the metric's trace acquires a delta-function singularity for a point source. (ii) Black mGR holes have hair-indeed their (spherically symmetric, static, conserved) spatial stresses affect the entire exterior metric $h_{i j}$, not just its trace in the FCI. The contact terms are enhanced by the square of mGR's range and are singular in the massless limit. (iii) The Newtonian potential now also depends-though it is highly damped —on the interior stresses.

\section{ACKNOWLEDGMENT}

We thank Bayram Tekin for useful discussions, and A. W. thanks the Perimeter Institute for hospitality while this research was completed. S. D. was supported in part by NSF Grant No. PHY-1064302 and DOE Grant No. DE-FG02-164 92ER40701.
[1] K. Hinterbichler, Rev. Mod. Phys. 84, 671 (2012).

[2] S. Deser and A. Waldron, Phys. Rev. Lett. 110, 111101 (2013); S. Deser, K. Izumi, Y. C. Ong, and A. Waldron, Phys. Lett. B 726, 544 (2013); arXiv:1312.1115; for a sampling of further mGR inconsistencies, see: A. Gruzinov, arXiv:1106.3972; C. Burrage, C. de Rham, L. Heisenberg, and A. J. Tolley, J. Cosmol. Astropart. Phys. 07 (2012) 004; P. de Fromont, C. de Rham, L. Heisenberg, A. Matas 
C.-I. Chiang, K. Izumi, and P. Chen, J. Cosmol. Astropart. Phys. 12 (2012) 025; E. Babichev and A. Fabbri, arXiv:1304.5992; A. De Felice, A. E. Gümrükçüoğlu, and S. Mukohyama, Phys. Rev. Lett. 109, 171101 (2012); A. De Felice, A. E. Gümrükçüoğlu, C. Lin, and S. Mukohyama, arXiv:1303.4154; arXiv:1304.0484; F. Kühnel, arXiv:1208.1764.

[3] These deviations are absent in the vector, Proca, analog of mGR. Consider a static source. The relevant equation, $\vec{\nabla} \cdot \vec{E}+m^{2} A_{0}=0 \Rightarrow\left(\Delta-m^{2}\right) A_{0}=\rho$ (the second equality holds because the erstwhile "gauge fixing," $\partial_{\mu} A^{\mu}=0$, is now a consequence of the Bianchi identity). In the present, static, case, this means that $\vec{\nabla} \cdot \vec{A}=0$, which excludes spherically symmetric $A_{i}$, leaving only the Yukawa equation for $A_{0}$; the electrostatic force is Yukawa, with no surprises.

[4] T. D. Lee and B. Zumino, Phys. Rev. 163, 1667 (1967).

[5] S. Deser and B.E. Laurent, Am. J. Phys. 36, 789 (1968).

[6] This universal assumption is, of course, not strictly consistent for FP since matter, being coupled to $h_{\mu \nu}$, is no longer isolated.

[7] B. Zumino, Brandeis University Lectures on Elementary Particles and Quantum Field Theory, Vol. 2, edited by S. Deser, M. Grisaru, and H. Pendleton, (MIT, Cambridge, MA, 1970), p. 437; C. de Rham, G. Gabadadze, and A. J. Tolley, Phys. Rev. Lett. 106, 231101 (2011); Phys. Lett. B 711, 190 (2012); C. de Rham and G. Gabadadze, Phys. Rev. D 82, 044020 (2010); S. F. Hassan and R. A. Rosen, Phys. Rev. Lett. 108, 041101 (2012).

[8] C. Deffayet, J. Mourad, and G. Zahariade, J. Cosmol. Astropart. Phys. 01 (2013) 032.

[9] S. Deser, M. Sandora, and A. Waldron, Phys. Rev. D 87, 101501 (2013).

[10] For one of the three allowed, five Dof, mass terms, a covariant scalar constraint is not known [8], but a noncovariant version of this result must hold.
[11] Absent spherical symmetry, $T_{i j}$ also admits transversetraceless components.

[12] In contrast, a slow GR probe (with only $t_{00}$ ) interacts with the interior [see Ref. [5] or Eq. (5)] according to $I \sim \int t_{00} C T$, where $C$ is the Coulomb potential. By virtue of Eq. (5), $T=\Delta \tau$ so $I \sim \int t_{00} C \Delta \tau=\int t_{00} \tau$, which (as promised) vanishes when the exterior $t_{00}$ and interior $\tau$ do not overlap.

[13] I. Gullu and B. Tekin, arXiv:1305.5461.

[14] The GR interaction formula $I=\int\left[t_{\mu \nu} C T^{\mu \nu}-\frac{1}{2} t_{\mu}^{\mu} C T_{\nu}^{\nu}\right]$ ostensibly involves all components $T_{\mu \nu}$, which seems to conflict with the absence of any long-range $T_{i j}$ effects and the "no hair" GR mantra that spherical exterior metrics only "see" $T_{00}$. The resolution is as follows. First, there is no $T_{0 i}$ hair because there exist no spherically symmetric transverse vectors/dipoles. Second, the interaction $I$ comes from the gravitational field, while $T_{\mu \nu}$ must separately obey locality and conservation constraints. Locality of $T_{\mu \nu}(x)$ is a physical requirement-its local values matter when they become sources of a field, unlike in special relativity, where only their space integrals, the Lorentz generators, or the total charge for currents $j_{\mu}(x)$, are physical (conservation is, of course, also required by the GR field's Bianchi identities). Locality and conservation of a time-independent source, such as a local tensor $T_{i j} \sim \delta_{i j} \delta^{3}(r)$, requires adding a long-range tail $\sim \partial_{i} \partial_{j}(1 / r)$. Reinstating locality entails multiplying this by a $\Delta$ factor. But then the resulting, ultralocal, $T_{i j} \sim\left(\delta_{i j} \Delta-\partial_{i} \partial_{j}\right) \delta^{3}(r)$ precludes long-range interactions - the extra $\Delta$ removes the Coulomb denominator (see Ref. [5]). An alternate explanation of GR's lack of $T_{i j}$ effects is that its counterpart of Eq. (6), namely, (up to a gauge) $h_{i j}=C\left(T_{i j}-\frac{1}{2} \delta_{i j} T\right)=-\frac{1}{2} C \partial_{i} \partial_{j} \tau(r)$. But this is precisely a gauge, hence irrelevant. The above considerations do not contradict the usual application of $I$ to light bending: Its Maxwell fields are time dependent, hence involve spacetime, rather than just space, conservation. 